\title{
Comparison of genomic predictions using genomic relationship matrices built with different weighting factors to account for locus-specific variances
}

\author{
G. Su, ${ }^{1}$ O. F. Christensen, L. Janss, and M. S. Lund \\ Center for Quantitative Genetics and Genomics, Department of Molecular Biology and Genetics, Aarhus University, DK-8830 Tjele, Denmark
}

\begin{abstract}
Various models have been used for genomic prediction. Bayesian variable selection models often predict more accurate genomic breeding values than genomic BLUP (GBLUP), but GBLUP is generally preferred for routine genomic evaluations because of low computational demand. The objective of this study was to achieve the benefits of both models using results from Bayesian models and genome-wide association studies as weights on single nucleotide polymorphism (SNP) markers when constructing the genomic matrix (G-matrix) for genomic prediction. The data comprised 5,221 progeny-tested bulls from the Nordic Holstein population. The animals were genotyped using the Illumina Bovine SNP50 BeadChip (Illumina Inc., San Diego, CA). Weighting factors in this investigation were the posterior SNP variance, the square of the posterior SNP effect, and the corresponding minus base-10 logarithm of the marker association $P$-value $\left[-\log _{10}(P)\right]$ of a $t$-test obtained from the analysis using a Bayesian mixture model with 4 normal distributions, the square of the estimated SNP effect, and the corresponding $\log _{10}(P)$ of a $t$-test obtained from the analysis using a classical genome-wide association study model (linear regression model). The weights were derived from the analysis based on data sets that were $0,1,3$, or 5 yr before performing genomic prediction. In building a G-matrix, the weights were assigned either to each marker (single-marker weighting) or to each group of approximately 5 to 150 markers (group-marker weighting). The analysis was carried out for milk yield, fat yield, protein yield, fertility, and mastitis. Deregressed proofs (DRP) were used as response variables to predict genomic estimated breeding values (GEBV). Averaging over the 5 traits, the Bayesian model led to $2.0 \%$ higher reliability of GEBV than the GBLUP model with an original unweighted G-matrix. The superiority of using a GBLUP with weighted G-matrix over GBLUP with an original unweighted G-matrix was the largest when
\end{abstract}

Received April 6, 2014.

Accepted July 7, 2014.

${ }^{1}$ Corresponding author: guosheng.su@agrsci.dk using a weighting factor of posterior variance, resulting in 1.7 percentage points higher reliability. The second best weighting factors were $-\log _{10}(P$-value $)$ of a $t$-test corresponding to the square of the posterior SNP effect from the Bayesian model and $-\log _{10}(P$-value) of a $t$-test corresponding to the square of the estimated SNP effect from the linear regression model, followed by the square of estimated SNP effect and the square of the posterior SNP effect. In addition, group-marker weighting performed better than single-marker weighting in terms of reducing bias of GEBV, and also slightly increased prediction reliability. The differences between weighting factors and scenarios were larger in prediction bias than in prediction accuracy. Finally, weights derived from a data set having a lag up to $3 \mathrm{yr}$ did not reduce reliability of GEBV. The results indicate that posterior SNP variance estimated from a Bayesian mixture model is a good alternative weighting factor, and common weights on group markers with a size of 30 markers is a good strategy when using markers of the 50,000-marker $(50 \mathrm{~K})$ chip. In a population with gradually increasing reference data, the weights can be updated once every $3 \mathrm{yr}$.

Key words: genomic relationship matrix, genomic selection, model, reliability

\section{INTRODUCTION}

Several statistical models have been proposed for genomic predictions using genome-wide SNP markers. One of the most popularly used models is genomic BLUP (GBLUP), which is a linear mixed model incorporating a marker-based genomic relationship matrix (G-matrix), because it is in the same form as a simple traditional BLUP model and has a low computational requirement. The G-matrix is built using the information of genome-wide dense markers (VanRaden, 2008; Hayes et al., 2009b). Compared with traditional pedigree-based models, it has the advantage of being able to capture linkage disequilibrium (LD) between markers and causal genes, Mendelian segregation, and genetic links through unknown common ancestors that are not available in the known pedigree. Therefore, the G-matrix is superior to the pedigree-based relationship 
matrix for genetic evaluation, and can be implemented in methods and models that conventionally incorporate a pedigree-based relationship matrix. In GBLUP models, the covariance matrix of additive genetic effect is generally defined to be proportional to the G-matrix under the assumption that given the same allele frequency, all the markers have the same contribution to the genetic variation of a trait. This is equivalent to assuming that the effects of all markers follow the same normal distribution (VanRaden, 2008; Strandén and Garrick, 2009). Obviously, the assumption is not desirable if the trait is affected by major genes.

Unlike GBLUP, Bayesian variable selection models allow variances of SNP effects differing among loci. This is usually realized by assuming a thick-tailed distribution of SNP effects or a mixture of 2 or more distributions (Meuwissen et al., 2001; Su et al., 2010; Habier et al., 2011; Erbe et al., 2012; Gao et al., 2013). Clearly, Bayesian variable selection models capture the feature of SNP effects better than GBLUP. Many simulation studies have shown that Bayesian models perform better than the GBLUP model (Meuwissen et al., 2001; Lund et al., 2009; Guo et al., 2010). Based on real cattle data, previous studies showed that Bayesian models led to similar or higher prediction accuracies than GBLUP (Hayes et al., 2009a; Habier et al., 2010; $\mathrm{Su}$ et al., 2012a). The benefit from Bayesian models is larger for traits controlled by large QTL (Cole et al., 2009; Legarra et al., 2011) and for animals that have weak relationship with individuals in the reference population (Habier et al., 2010; Gao et al., 2013). However, typical Bayesian variable selection models using the Markov chain Monte Carlo (MCMC) algorithm have the disadvantage of a long computing time.

Because in a GBLUP model the covariance matrix is proportional to the G-matrix, a simple way to overcome the disadvantage of the inappropriate assumptions of an original GBLUP model is to build a G-matrix in which markers are weighted appropriately. Zhang et al. (2010) proposed a method to build a trait-specific G-matrix for genomic prediction using GBLUP and analyzed simulation data. They reported that the accuracy of genomic EBV (GEBV) increased when using a GBLUP model with a G-matrix weighted by squared marker effect estimated from a random regression BLUP model, compared with the original GBLUP. The accuracies further increased when using a G-matrix weighted with the posterior variance of the marker effect estimated from a BayesB model. Based on data from French Holstein and Montbéliarde cattle, Legarra et al. (2011) predicted genomic breeding values using a GBLUP with a G-matrix weighted by the posterior variance of the marker effect derived from the analysis using a Bayesian least absolute shrinkage and selec- tion operator (LASSO) model, and obtained prediction accuracies close to those using the Bayesian LASSO directly. A study by de los Campos et al. (2013) used the minus base-10 logarithm of the marker association $P$-value $\left[-\log _{10}(P)\right]$ from a genome-wide association study (GWAS) as weight on the makers to build a G-matrix, and reported that the weighted G-matrix improved prediction accuracy, based on human type-2 diabetes case-control data sets. In contrast, Zhou et al. (2014) reported that a G-matrix weighted with the square of estimated marker effect led to lower accuracy of genomic prediction than an original G-matrix, based on the Nordic dairy cattle data.

It can be hypothesized that an appropriately weighted G-matrix can improve the prediction reliability of a GBLUP model, and a GBLUP model with a G-matrix weighted using the posterior variance of the marker effect from a Bayesian variable selection model can achieve the similar prediction reliability as the Bayesian variable selection model. The objective of this study was to test these hypotheses by assessing alternative weighting factors to construct weighted G-matrices for genomic prediction. In addition, this study investigated the ways to weight markers, and the time intervals when weights need to be updated. The analysis was based on data from the Nordic Holstein population.

\section{MATERIALS AND METHODS}

\section{Data}

The data in this analysis comprised 5,643 progenytested bulls from the Nordic Holstein population. The animals were genotyped with the Illumina Bovine SNP50 BeadChip (Illumina Inc., San Diego, CA; Matukumalli et al., 2009). The marker data were edited by deleting markers with minor allele frequency lower than 0.01 , average GenCall score lower than 0.60 , or unknown location in the UMD 3.1 assembly [University of Maryland, College Park]. After editing, 44,919 markers remained in the analysis. Sporadic missing genotypes were imputed using the BEAGLE software package (Browning and Browning, 2009). The phenotypic data for genomic prediction were deregressed proofs (DRP), which were derived from the Nordic genetic evaluations in January 2013. The traits in the analysis were milk yield, fat yield, protein yield, fertility, and mastitis.

The data were divided into a reference data set and a validation data set by birth date (January 1, 2005). This resulted in about the $20 \%$ youngest bulls being validation bulls. Deregressed proofs with reliability less than $10 \%$ were excluded from the reference data and less than $20 \%$ were removed from the test data. The number of animals with phenotypic information dif- 
Table 1. Number of bulls in reference (Ref) and validation data sets

\begin{tabular}{lccccc}
\hline Trait & $\operatorname{Ref}_{\mathrm{w} 2000}{ }^{1}$ & $\operatorname{Ref}_{\mathrm{w} 2002}{ }^{1}$ & $\operatorname{Ref}_{\mathrm{w} 2004}{ }^{1}$ & $\operatorname{Ref}^{2}$ & Validation \\
\hline Milk & 2,464 & 3,030 & 3,616 & 3,949 & 1,159 \\
Fat & 2,464 & 3,030 & 3,616 & 3,949 & 1,159 \\
Protein & 2,464 & 3,030 & 3,616 & 3,949 & 1,159 \\
Fertility & 2,500 & 3,064 & 3,648 & 3,975 & 1,158 \\
Mastitis & 2,505 & 3,073 & 3,660 & 3,987 & 1,204 \\
\hline
\end{tabular}

${ }^{1}$ The Ref population (bulls born before 2000, 2002, or 2004) was used for deriving weighting (w) factors only.

${ }^{2}$ The Ref population (bulls born in 2005) was used for both deriving weighting factors and predicting genomic EBV.

fered among different traits due to different numbers of published EBV available. In total, 5,221 bulls remained in the analysis. The numbers of animals available in genomic prediction for different traits are shown in Table 1.

\section{Statistical Models}

Genomic predictions were performed using GBLUP models with unweighted G-matrix, Bayesian mixture models, and GBLUP models with the G-matrices built using different weighting factors. These models are described below.

GBLUP Model. The model with unweighted Gmatrix is

$$
\mathbf{y}=\mathbf{1} \mu+\mathbf{Z g}+\mathbf{e}
$$

where $\mathbf{y}$ is the vector of DRP of genotyped reference bulls, $\mu$ is the overall mean, $\mathbf{g}$ is the vector of additive genetic effects for the genotyped bulls, $\mathbf{e}$ is the vector of random residuals, $\mathbf{1}$ is the vector with all elements equal to 1 , and $\mathbf{Z}$ is the design matrix associating $\mathbf{g}$ with response variables.

The distribution of residual effects is assumed to be $\mathbf{e} \sim \mathrm{N}\left(\mathbf{0}, \mathbf{R} \sigma_{e}^{2}\right)$, where $\sigma_{e}^{2}$ is the residual variance, and $\mathbf{R}$ is a diagonal matrix with elements $\mathrm{R}_{\mathrm{ii}}=\left(1-\mathrm{r}_{\mathrm{DRP}}^{2}\right) / \mathrm{r}_{\mathrm{DRP}}^{2}$ (VanRaden, 2008; Garrick et al., 2009), where $\mathrm{r}_{\mathrm{DRP}}^{2}$ is the reliability of DRP; $R_{\mathrm{ii}}$ was applied to account for heterogeneous residual variances due to different reliabilities of $\operatorname{DRP}\left(\mathrm{r}_{\mathrm{DRP}}^{2}\right)$. It is assumed that $\mathbf{g} \sim \mathrm{N}\left(\mathbf{0}, \mathbf{G} \sigma_{g}^{2}\right)$, where $\sigma_{g}^{2}$ is the additive genetic variance and $\mathbf{G}$ is the realized genomic relationship matrix, calculated from marker genotypes without using pedigree information. Following VanRaden (2008),

$$
\mathbf{G}=\mathbf{M D M}^{\prime} / n_{m},
$$

where $n_{m}$ is the number of markers; elements in column $j\left(m_{i j}\right)$ of $\mathbf{M}$ are $0-2 p_{j}, 1-2 p_{j}$, and $2-2 p_{j}$ for genotypes $A_{1} A_{1}, A_{1} A_{2}$, and $A_{2} A_{2}$, respectively, where $p_{j}$ is the allele frequency of $\mathrm{A}_{2}$ at locus $j$ calculated from the current marker data; and $\mathbf{D}$ is a diagonal matrix for scaling $\mathbf{G}$ to be analogous to the conventional pedigreebased numerator relationship matrix. Two scaling factors have been defined. One is a constant scale for all markers [method 1 in VanRaden (2008)]:

$$
d_{j j}=\frac{1}{\sum_{j=1}^{n_{m}} 2 p_{j}\left(1-p_{j}\right) / n_{m}}
$$

(denoting the corresponding G-matrix as G-matrix1 in the context), and the other is locus specific (method 2 in VanRaden, 2008):

$$
d_{j j}=\frac{1}{2 p_{j}\left(1-p_{j}\right)}
$$

(denoting the G-matrix as G-matrix2).

GBLUP Model with Weighted G-Matrix. The model is the same as the above GBLUP model except for the G-matrix, which is calculated as

$$
\mathbf{G}_{\mathbf{w}}=\mathbf{M D}^{*} \mathbf{M}^{\prime} / n_{m},
$$

where $\mathbf{G}_{\mathbf{w}}=$ the weighted G-matrix, $\mathbf{D}^{*}=\mathbf{D T}$, and $\mathbf{T}$ is a diagonal matrix with diagonal element $j$ being the weight on the marker at locus $j$ (see definition of weight in the next section).

Bayesian Mixture Model with 4 Normal Distributions. The model is

$$
\mathbf{y}=\mathbf{1} \mu+\mathbf{X q}+\mathbf{e}
$$

where $\mathbf{X}$ is the matrix of marker genotype coefficients and $\mathbf{q}$ is the vector of SNP effects. It can be written $\mathbf{X}$ $=\mathbf{M D}^{0.5}$, where $\mathbf{M}$ is as defined above, and $\mathbf{D}^{0.5}$ is a diagonal matrix with diagonal element $d_{j j}^{0.5}$ equal to the square root of $d_{j j}$ in the matrix $\mathbf{D}$ defined above. Similar to the 2 scale matrices for G-matrix, $\mathbf{D}^{0.5}$ can have 
2 forms. The first form is $d_{j j}^{0.5}=1 / \sqrt{\sum_{j=1}^{n_{m}} 2 p_{j}\left(1-p_{j}\right) / n_{m}}$. In this form, $d_{j j}^{0.5}$ is a constant that has no influence on prediction; therefore, $\mathbf{M} \mathbf{D}^{0.5}$ can be replaced by $\mathbf{M}$ (i.e., $\mathbf{X}=\mathbf{M}$; Habier et al., 2011). The second form is $d_{j j}^{0.5}=1 / \sqrt{2 p_{j}\left(1-p_{j}\right)}$ (Meuwissen and Goddard, 2010). The model assumes that the distribution of marker effects is a mixture of 4 normal distributions:

$$
q_{j} \sim \pi_{1} N\left(0, \sigma_{1}^{2}\right)+\pi_{2} N\left(0, \sigma_{2}^{2}\right)+\pi_{3} N\left(0, \sigma_{3}^{2}\right)+\pi_{4} N\left(0, \sigma_{4}^{2}\right),
$$

where $\pi_{i}$ is the mixing proportion and $\sigma_{i}^{2}$ is the variance of distribution $i$. For the model with $\mathbf{X}=\mathbf{M}$ (denoted as $\mathbf{X}$-matrix $\mathbf{1}), \sigma_{i}^{2}$ is the variance of a SNP effect. For the model with $\mathbf{X}=\mathbf{M D}^{0.5}$ and $d_{j j}^{0.5}=1 / \sqrt{2 p_{j}\left(1-p_{j}\right)}$ (denoted as $\mathbf{X}$-matrix 2 ), $\sigma_{i}^{2}$ is the population variance explained by a SNP. In theory, both $\pi_{i}$ and variances can be estimated simultaneously from data. However, this strategy often results in poor mixing properties of the MCMC algorithm. Therefore in the present study, the mixing proportions averaged over loci were taken as known and set to $\pi_{1}=0.889, \pi_{2}=0.1, \pi_{3}=0.01$, and $\pi_{4}=0.001$, and the variances were estimated under the constraint $\sigma_{1}^{2}<\sigma_{2}^{2}<\sigma_{3}^{2}<\sigma_{4}^{2}$. The mixture model is similar to BayesR (Erbe et al., 2012). The difference is that the present mixture model allows a small $\sigma_{1}^{2}$, whereas BayesR sets $\sigma_{1}^{2}$ to be zero. The MCMC implementation is straightforward with recognizable conditional distributions for all model parameters and the indicator variable assigning each SNP to 1 of the 4 distributions (George and McCulloch, 1993).

In addition to the models above, another model used in this study was a linear model with a single SNP fixed regression (i.e., a typical genome-wide association analysis model, denoted as a GWAS model in the following context). This model was only used to derive alternative weighting factors to build the weighted Gmatrix. The GWAS model is

$$
\mathbf{y}=\mathbf{1} \mu+\mathbf{m b}+\mathbf{Z a}+\mathbf{e},
$$

where $\mathrm{b}$ is the fixed regression coefficient (i.e., SNP effect) of a SNP, $\mathbf{m}$ is the vector of genotypes of individuals at this SNP, $\mathbf{a}$ is the vector of additive genetic effects, and $\mathbf{Z}$ is the design matrix associating a with response variables. It is assumed that $\mathbf{a} \sim \mathrm{N}\left(\mathbf{0}, \mathbf{A} \sigma_{a}^{2}\right)$, where $\mathbf{A}$ is the pedigree-based relationship matrix and $\sigma_{a}^{2}$ is the additive genetic variance, which is equal to $\sigma_{g}^{2}$ in the GBLUP model if SNP markers can account for all additive genetic variance. Using this model, SNP effects are estimated separately, 1 SNP at a time.

\section{Posterior Variances from the Bayesian Mixture Model as Weighting Factors to Build the G-Matrix}

Using the Bayesian mixture models, genomic breeding value is defined as $\mathbf{g}=\mathbf{X q}$. For the model with $\mathbf{X}$ $=\mathbf{M D}^{0.5}, \mathbf{g}=\mathbf{M D}^{0.5} \mathbf{q}$, the covariance matrix of $\mathbf{g}$ is

$$
\begin{aligned}
& \operatorname{Var}(\mathbf{g})=\mathbf{M D}^{0.5} \mathbf{V}_{\mathbf{q}} \mathbf{D}^{0.5 \prime} \mathbf{M}^{\prime}=\frac{\mathbf{M D}^{0.5} \mathbf{V}_{\mathbf{q}} \mathbf{D}^{0.5 /} \mathbf{M}^{\prime} n_{m} \bar{v}_{q}}{n_{m} \bar{v}_{q}} \\
& =\frac{\mathbf{M D T M}^{\prime} \sigma_{g}^{2}}{n_{m}}=\mathbf{G}_{\mathbf{w}} \sigma_{g}^{2} .
\end{aligned}
$$

In this equation, $\mathbf{V}_{\mathbf{q}}$ is the variance matrix with the $j$ th diagonal element being $v_{a j}$, which is the variance explained by SNP $j$ and all off-diagonals being zero; $\mathbf{T}$ is a diagonal matrix and its $j$ th diagonal element (i.e., weight for marker $j$ ) is $t_{j j}=v_{q j} / \bar{v}_{q}$, where $\bar{v}_{q}=\sum_{j=1}^{n_{m}} v_{q j} / n_{m}$ is the mean of $v_{g j}$; and $\sigma_{g}^{2}=\sum_{j=1}^{n_{m}} v_{q j}=n_{m} \bar{v}_{q}$ is the additive genetic variance. It should be noted that matrix $\mathbf{D}$ for the G-matrix $\left(\mathbf{G}_{\mathrm{w}}=\mathbf{M D T M}^{\prime}\right)$ must be consistent with $\mathbf{D}^{0.5}$ in the Bayesian mixture. For example, when using $\mathbf{D}^{0.5}$ with $d_{j j}^{0.5}=1 / \sqrt{2 p_{j}\left(1-p_{j}\right)}$ in the Bayesian mixture model, the diagonal of $\mathbf{D}$ for the $\mathbf{G}_{\mathbf{w}}$ must be $d_{j j}=1 / 2 p_{j}\left(1-p_{j}\right)$.

For the Bayesian mixture model with $\mathbf{X}=\mathbf{M}$, the corresponding covariance matrix $\operatorname{Var}(\mathbf{g})$ is

$$
\begin{aligned}
& \operatorname{Var}(\mathbf{g})=\mathbf{M V}_{\mathbf{q}} \mathbf{M}^{\prime}=\frac{\mathbf{M D V}_{\mathbf{q}} \mathbf{M}^{\prime} \sum_{j=1}^{n_{m}} 2 p_{j}\left(1-p_{j}\right) \bar{v}_{q}}{n_{m} \bar{v}_{q}} \\
& =\frac{\mathbf{M D T M}^{\prime} \sigma_{g}^{2}}{n_{m}}=\mathbf{G}_{\mathbf{w}} \sigma_{g}^{2},
\end{aligned}
$$

where

$$
d_{j j}=\frac{1}{\sum_{j=1}^{n_{m}} 2 p_{j}\left(1-p_{j}\right) / n_{m}}
$$

and $t_{j j}=v_{q j} / \bar{v}_{q}$. In such case, the additive genetic variance is $\sigma_{g}^{2}=\sum_{j=1}^{n_{m}} 2 p_{j}\left(1-p_{j}\right) \bar{v}_{q}$.

The Bayesian models with different forms of matrix $\mathbf{X}$ can result in their equivalent covariance matrix to be 
Table 2. Reliability of genomic EBV (GEBV) and regression coefficient of deregressed proof on GEBV, obtained from different models ${ }^{1}$

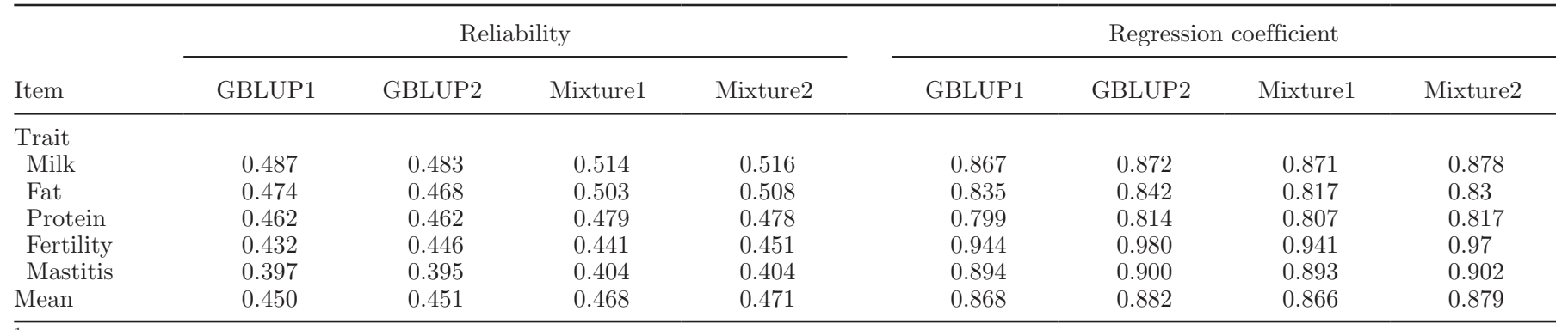

${ }^{1}$ GBLUP1 = GBLUP model with a genomic relationship matrix (G-matrix) scaled by average $2 p(1-p)$, where $p$ is the allele frequency of the second allele of a SNP locus; GBLUP2 = GBLUP model with a G-matrix scaled by locus-specific $2 p(1-\mathrm{p})$; Mixture1 = Bayesian mixture model with a design matrix of genotypes that was not scaled; Mixture $2=$ Bayesian mixture model with a design matrix of genotypes that was scaled by locus-specific $2 p(1-p)$.

used in a GBLUP model. In this study, the Bayesian model with $\mathbf{X}=\mathbf{M D}^{0.5}$ and $d_{j j}^{0.5}=1 / \sqrt{2 p_{j}\left(1-p_{j}\right)}$ was chosen to derive weighting factors, as this model performed slightly better than the Bayesian model with $\mathbf{X}$ $=\mathbf{M}$, based on the present data (Table 2).

As shown above, a G-BLUP model with a G-matrix weighted by $v_{q j} / \bar{v}_{q}$ is equivalent to the corresponding Bayesian model. However, $v_{q j} / \bar{v}_{q}$ is unknown. An approach is to use the posterior variance from the Bayesian mixture model as weighting factor $\left(\mathbf{W V}_{\mathbf{q}}\right)$, calculated as $\hat{v}_{q j} / \overline{\hat{v}}_{q}=\sum_{i=1}^{4} \hat{\pi}_{i} \hat{\sigma}_{i}^{2} / \overline{\hat{v}}_{q}$, where $\hat{\pi}_{i}$ is the posterior probability for SNP $j$ and $\hat{\sigma}_{i}^{2}$ is the posterior variance of the $i$ th distribution in the mixture model, and $\overline{\hat{v}}=\sum_{j=1}^{n_{m}} \sum_{i=1}^{4} \hat{\pi}_{i} \hat{\sigma}_{i}^{2} / n_{m}=\sum_{j=1}^{n_{m}} \hat{v}_{q j} / n_{m}$ is the mean of $\hat{v}_{q j}$.

\section{Alternative Weighting Factors}

Besides $\mathrm{WV}_{\mathrm{q}}, 4$ alternative weighting factors were also investigated in this study. These weighting factors are described as follows.

Square of the Posterior SNP Effect. The square of the posterior SNP effect $\left(\mathbf{W q}^{2}\right)$ is the square of SNP effect estimated from the Bayesian mixture model, calculated as $\hat{q}_{j}^{2} / \overline{\hat{q}}^{2}$, where $\overline{\hat{q}}^{2}$ is the mean of $\hat{q}_{j}^{2}$.

Corresponding - $\log _{10}\left(P_{q j}\right)$ from a $t$-Test Using a Bayesian Mixture Model. The corresponding $\log _{10}\left(P_{q j}\right)$ from a $t$-test $\left(\mathbf{W P}_{\mathbf{q}}\right)$ for SNP effects $\left(\hat{q}_{j}\right)$ is estimated from the Bayesian mixture model divided by the mean of $-\log _{10}\left(P_{q j}\right) \quad$ \{i.e., $-\log _{10}\left(P_{q j}\right) /$ mean $[-$ $\left.\left.\log _{10}\left(P_{q j}\right)\right]\right\}$.

Square of the Estimated SNP Effect. The square of the estimated SNP effect $\left(\hat{b}_{j}^{2}\right)$ is estimated from the GWAS $\left(\mathbf{W b}^{\mathbf{2}}\right)$. Because the vector of genotypes in the GWAS model was not scaled by $\frac{1}{2 p_{j}\left(1-p_{j}\right)}$, to keep consistent with the G-matrix scaled by $\frac{1}{2 p_{j}\left(1-p_{j}\right)}$, the weight was calculated as $2 p_{j}\left(1-p_{j}\right) \hat{b}_{j}^{2}$ divided by the mean of $2 p_{j}\left(1-p_{j}\right) \hat{b}_{j}^{2}$.

Corresponding $-\log _{10}\left(P_{b j}\right)$ from a $t$-Test $U s$ ing a $\boldsymbol{G} \boldsymbol{W A S}$ Model. The corresponding $-\log _{10}\left(P_{b j}\right)$ from a $t$-test $\left(\mathbf{W P}_{\mathbf{b}}\right)$ for SNP effects is estimated from the GWAS model, calculated as $-2 p_{j}\left(1-p_{j}\right) \log _{10}\left(P_{b j}\right)$ divided by the mean of $-2 p_{j}\left(1-p_{j}\right) \log _{10}\left(P_{b j}\right)$.

\section{Single-Marker Weighting, Group-Marker Weighting, and Analysis with Different Data Sets and Models}

In addition to exerting a weight on an individual marker (denoted as single-marker weighting in the following context), this study also used a common weight for markers on a chromosomal region (denoted as group-marker weighting) with a length of $5,10,30$, 50, 70, 100, and 150 markers. The common weight was calculated as the mean of individual weights over the markers in the same group.

Four data sets were used to derive weights to build the G-matrix for genomic prediction. The first data set was the full reference data set (bulls born before 2005) used for final genomic prediction. The second consisted of bulls born before 2004, the third before 2002, and the fourth before 2000. The scenarios of using these reduced data sets to derive weights were performed to assess the influence of a lag in updating weighting factors on genomic prediction.

Genomic breeding values were predicted using the Bayesian mixture models, the GBLUP models with the original G-matrix, and weighted G-matrices. When using GBLUP models with a weighted G-matrix for genomic prediction, the additive genetic variance and residual variance were obtained from the analysis using the Bayesian mixture model based on the corresponding data. The additive genetic variance was calculated as 
$\sum_{j=i}^{n_{m}} \sum_{i=1}^{4} \hat{\pi}_{i} \hat{v}_{i}$, where $\hat{\pi}_{i}$ is the posterior probability across SNP loci and $\hat{v}_{i}$ is the posterior variance of the ith distribution in the mixture model.

The analysis using the GBLUP models and the GWAS model were performed using the DMU package (Madsen et al., 2010). The analysis using the Bayesian models were performed using the Bayz package (http:// www.bayz.biz).

\section{Validation}

Genomic predictions using different models were validated by comparing GEBV with DRP for the validation bulls. Reliability of genomic prediction was measured as the squared correlations between predicted breeding values and DRP and then divided by mean reliability of DRP (Su et al., 2012b). Unbiasedness of genomic predictions was measured as the regression of DRP on the genomic predictions (Su et al., 2012a).

\section{RESULTS}

\section{Genomic Predictions Using the Original GBLUP Models and Bayesian Mixture Models}

As shown in Table 2, the reliabilities of GEBV ranged from 0.395 to 0.487 using the GBLUP models. The 2 GBLUP models led to similar reliability of GEBV for 4 traits, but for fertility, the GBLUP with G-matrix2 (GBLUP2) performed better than the one with Gmatrix1 (GBLUP1). The reliabilities of GEBV were between 0.404 and 0.516 using the Bayesian mixture models. Similar to the GBLUP models, the 2 Bayesian models resulted in similar reliability of GEBV for most of the traits, except for fertility in which the Bayesian mixture model with X-matrix2 (Mixture2) had higher prediction reliability than the model with $\mathrm{X}$-matrix1
(Mixture1). Averaged over the 5 traits, the reliability of GEBV when using the Mixture1 was $1.7 \%$ points higher than that when using GBLUP1, and Mixture2 was $2.0 \%$ higher than GBLUP2. The gain from the Bayesian model was largest for fat and milk.

For all models, an inflation of GEBV occurred, as indicated by the regression coefficients being less than 1 for all traits. The inflation was more serious for the 3 production traits (especially protein and fat) than for mastitis and fertility. The regression coefficients of DRP on GEBV were closer to 1 when using GBLUP2 and Mixture2 than GBLUP1 and Mixture1. The differences in regression coefficients were very small between GBLUP1 and Mixture1 and between GBLUP2 and Mixture2.

\section{Genomic Predictions Using the GBLUP Models with a Weighted G-Matrix}

Table 3 presents reliabilities of genomic predictions, averaged over the 5 traits. The weighted G-matrices gave more accurate predictions than the original Gmatrix, except for the G-matrices weighted by $\mathrm{Wq}^{2}$, which resulted in lower reliabilities of GEBV when using single-marker weighting and using group-marker weighting with a group size of 5 . The highest reliabilities were obtained when using $\mathrm{WV}_{\mathrm{q}}$ and lowest when using $\mathrm{Wq}^{2}$.

The effect of weighting methods (single-marker weighting and group-marker weightings with various group sizes) on prediction reliability was large when using $\mathrm{Wq}^{2}$ but small using the other weighting factors. In terms of reliability of GEBV, the optimal group size for group-marker weighting was 30 markers. In this scenario, the GBLUP model with a G-matrix weighted by $\mathrm{WV}_{\mathrm{q}}$ resulted in $1.7 \%$ higher reliability of GEBV than the original GBLUP model, averaged over 5 traits. The gains from other weighted G-matrices were $0.9 \%$ when

Table 3. Reliability of genomic prediction using genomic BLUP (GBLUP) with a genomic relationship matrix (G-matrix) weighted by different weighting factors, using single-marker weighting (M_1) and group-marker weighting with group sizes ranging from 5 to 150 markers (M_5-M_150) $)^{1}$

\begin{tabular}{lccccccccc}
\hline Item & M_1 & M_5 & M_10 & M_30 & M_50 & M_70 & M_100 & M_150 & Mean \\
\hline Weight $^{2}$ & & & & & & & & \\
$W^{2}$ & 0.464 & 0.465 & 0.467 & 0.468 & 0.467 & 0.466 & 0.466 & 0.464 \\
Wq $^{2}$ & 0.446 & 0.448 & 0.453 & 0.456 & 0.456 & 0.451 & 0.454 & 0.453 \\
PP $_{\mathrm{q}}$ & 0.457 & 0.458 & 0.459 & 0.460 & 0.460 & 0.460 & 0.459 & 0.457 \\
Wb $^{2}$ & 0.456 & 0.456 & 0.457 & 0.457 & 0.456 & 0.456 & 0.455 & 0.455 \\
WP $_{\mathrm{b}}$ & 0.458 & 0.458 & 0.458 & 0.460 & 0.458 & 0.458 & 0.458 & 0.456 \\
Mean & 0.456 & 0.457 & 0.459 & 0.460 & 0.459 & 0.458 & 0.458 & 0.457 \\
\hline
\end{tabular}

${ }^{1}$ The reliability was on the average of 5 traits.

${ }^{2} \mathrm{WV}_{\mathrm{q}}=$ posterior variance of individual marker effect from the Bayesian mixture model; Wq ${ }^{2}=$ square of the marker effect estimated from the Bayesian mixture model; $\mathrm{WP}_{\mathrm{q}}=-\log _{10}(P$-value $)$ from a $t$-test for $\mathrm{SNP}$ effect estimated from the Bayesian mixture model; Wb ${ }^{2}=\mathrm{square}$ of marker effect estimated from the genome-wide association study (GWAS) model; $\mathrm{WP}_{\mathrm{b}}=-\log _{10}(P$-value) from a $t$-test for SNP effect estimated from the GWAS model. 
Table 4. Regression coefficient of deregressed proof on genomic prediction using genomic BLUP (GBLUP) with a genomic relationship matrix (G-matrix) weighted by different weighting factors, using single-marker weighting (M_1) and group-marker weighting with group sizes ranged from 5 to 150 markers (M_5-M_150) ${ }^{1}$

\begin{tabular}{lccccccccc}
\hline Item & M_1 & M_5 & M_10 & M_30 & M_50 & M_70 & M_100 & M_150 & Mean \\
\hline Weight $^{2}$ & & & & & & & & \\
WV $^{q}$ & 0.832 & 0.860 & 0.871 & 0.878 & 0.880 & 0.881 & 0.882 & 0.886 \\
Wq $^{2}$ & 0.761 & 0.804 & 0.826 & 0.852 & 0.867 & 0.868 & 0.870 & 0.873 \\
WP $_{q}$ & 0.822 & 0.858 & 0.869 & 0.879 & 0.882 & 0.884 & 0.884 & 0.885 \\
Wb $^{2}$ & 0.838 & 0.852 & 0.857 & 0.862 & 0.863 & 0.866 & 0.865 & 0.867 & 0.840 \\
WP $_{\mathrm{b}}$ & 0.837 & 0.852 & 0.859 & 0.866 & 0.866 & 0.869 & 0.869 & 0.871 \\
Mean & 0.818 & 0.845 & 0.856 & 0.867 & 0.872 & 0.874 & 0.874 & 0.876 \\
\hline
\end{tabular}

${ }^{1}$ The regression coefficient was on the average of 5 traits.

${ }^{2} \mathrm{WV}_{\mathrm{q}}=$ posterior variance of individual marker effect from the Bayesian mixture model; $\mathrm{Wq}^{2}=$ square of the marker effect estimated from the Bayesian mixture model; $\mathrm{WP}_{\mathrm{q}}=-\log _{10}(P$-value $)$ from a $t$-test for SNP effect estimated from the Bayesian mixture model; $\mathrm{Wb}^{2}=\mathrm{square}$ of marker effect estimated from the genome-wide association study (GWAS) model; $\mathrm{WP}_{\mathrm{b}}=-\log _{10}(P$-value) from a $t$-test for SNP effect estimated from the GWAS model.

using $\mathrm{WP}_{\mathrm{q}}$ and $\mathrm{WP}_{\mathrm{b}}, 0.6 \%$ when using $\mathrm{Wb}^{2}$, and $0.5 \%$ when using $\mathrm{Wq}^{2}$.

Increasing the group size resulted in less biased predictions for all weighting factors (Table 4). When single-marker weights were used, all weighting factors resulted in a regression coefficient much lower than the GBLUP with the original G-matrix and the Bayesian model. The effect was most serious when using $\mathrm{Wq}^{2}$ as weights. The regression coefficients increased largely with increasing group size up to 30 markers (50 markers for $\mathrm{Wq}^{2}$ ), and thereafter the increases were small. When using $\mathrm{WV}_{\mathrm{q}}$ or $\mathrm{WP}_{\mathrm{q}}$ and applying group weighting with a size of 30 markers, the regression coefficients were similar to those using the GBLUP with the original G-matrix and the Bayesian model. For other weighting factors, the regression coefficients were still lower than those from the original GBLUP and Bayesian models.

\section{Effect of a Lag in Updating Weights on Genomic Predictions}

The effect of a lag in updating weights on genomic prediction was investigated for the best weighting factor (i.e., $\mathrm{WV}_{\mathrm{q}}$ ) in this study. This was done by validat- ing genomic predictions using the G-matrices that were built with weights derived from old data sets. As can be seen in Table 5 , on average, over 5 traits, weights derived from a data set having a lag of 5 yr slightly reduced the reliability of GEBV, whereas a lag up to $3 \mathrm{yr}$ did not reduce reliability of GEBV. However, looking into individual traits, slight differences existed in the reliabilities of genomic predictions between the data sets.

Similar to the reliability of GEBV, the regression coefficients of DRP on GEBV were similar when using weights derived from different data sets, on average, over 5 traits (Table 6). However, a relative large difference was observed in milk yield for single-marker weighting and in fertility for both single-marker and group-marker weightings. Based on any of the 4 data sets, group-marker weighting had less bias than singlemarker weighting.

\section{DISCUSSION}

The present study compared genomic prediction using G-matrices weighted with various weighting factors. The results indicate that GBLUP models can

Table 5. Reliability of genomic prediction using the genomic BLUP (GBLUP) model with a genomic relationship matrix (G-matrix) weighted by posterior variance of individual marker effect ${ }^{1}$

\begin{tabular}{|c|c|c|c|c|c|c|c|c|}
\hline \multirow[b]{2}{*}{ Item } & \multicolumn{4}{|c|}{ Single-marker weighting } & \multicolumn{4}{|c|}{ Group-marker weighting with group size of 30 markers } \\
\hline & Lag 0 yr & Lag 1 yr & Lag 3 yr & Lag 5 yr & Lag 0 yr & Lag 1 yr & Lag 3 yr & Lag 5 yr \\
\hline Milk & 0.502 & 0.501 & 0.502 & 0.501 & 0.511 & 0.511 & 0.508 & 0.506 \\
\hline Fat & 0.491 & 0.489 & 0.494 & 0.487 & 0.505 & 0.505 & 0.508 & 0.499 \\
\hline Protein & 0.477 & 0.473 & 0.474 & 0.471 & 0.472 & 0.470 & 0.469 & 0.467 \\
\hline Fertility & 0.449 & 0.452 & 0.447 & 0.445 & 0.449 & 0.448 & 0.446 & 0.445 \\
\hline
\end{tabular}

${ }^{1}$ The posterior variance was estimated from the Bayesian mixture model and based on the data set that was $0,1,3$, or 5 yr before the genomic prediction. 
Table 6. Regression coefficient of a deregressed proof on genomic prediction using genomic BLUP (GBLUP) with a genomic relationship matrix (G-matrix) weighted by posterior variance of individual marker effect ${ }^{1}$

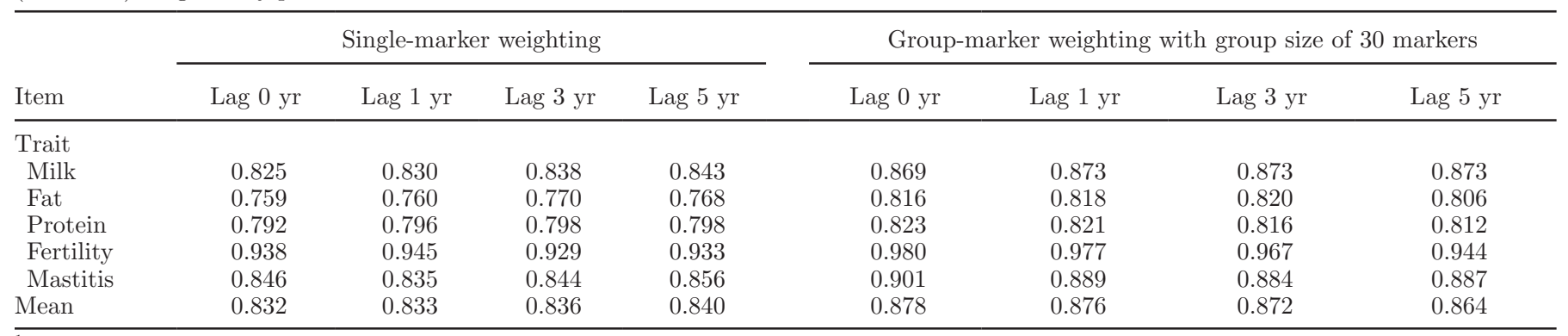

${ }^{1}$ The posterior variance was estimated from the Bayesian mixture model and based on the data set that was $0,1,3$, or 5 yr before the genomic prediction.

achieve prediction reliabilities close to those obtained from Bayesian variable selection models, by building a G-matrix weighted with appropriate weighting factors. The gains from weighted G-matrices are different when using different weighting factors and weighting methods.

\section{Comparison on Different Weighting Factors}

Many previous studies have reported that a weighted G-matrix leads to better genomic predictions than an original G-matrix (Zhang et al., 2010; Legarra et al., 2011; de los Campos et al., 2013). In this study, 5 weighting factors derived from 2 models were used to weight markers in the construction of a G-matrix. Weighting factor $\mathrm{WV}_{\mathrm{q}}$ was chosen because the resulting G-matrix was consistent with the covariance matrix of a Bayesian mixture model. The other weighting factors were used because they reflected the relative contributions of the markers to the genetic variation of the trait and could improve genomic predictions (Zhang et al., 2010; de los Campos et al., 2013). The Bayesian mixture model was chosen to derive weighting factors, as previous study on the Nordic Holstein population showed that this model outperformed the other models in the comparison (Gao et al., 2013). The GWAS model was considered because promising results were observed in the study by de los Campos et al. (2013).

In the current study, GBLUP with a weighted Gmatrix, in general, resulted in higher prediction accuracy than the original G-matrix. However, the G-matrix weighted with $\mathrm{Wq}^{2}$ using single-marker weighting resulted in lower reliabilities than the original G-matrix. Similarly, Zhou et al. (2014) reported that a G-matrix weighted with the square of the estimated marker effect from a random regression BLUP model led to lower accuracy of genomic prediction than the original Gmatrix, based on the Nordic dairy cattle data. Zhang et al. (2010) reported that the posterior variance of marker effect from BayesB as a weighting factor out- performed the square of marker effect from a random regression BLUP model as weighting factor. Unlike the genomic prediction model, where effects of all SNP are estimated simultaneously, the GWAS model estimates the effect of 1 SNP at each run. Thus, the sum of estimated SNP effects overestimates the QTL effects because several linked markers all estimate the effect of the same QTL. Correspondingly, the sum of SNP variances defined as $2 p_{j}\left(1-p_{j}\right) \hat{b}_{j}^{2}$ would be greatly larger than the total additive genetic variance. However, by standardizing the weight to a mean weight of 1 , the overestimation is no longer a problem when using the results from the GWAS as weighting factors. In line with de los Compos et al. (2013), a G-matrix weighted with $-\log _{10}(P)$ for marker association from the GWAS model resulted in a clear increase in prediction reliability. As expected, the best weighting factor in the current study was the posterior variance of marker effect from the Bayesian mixture model. This indicates that the posterior variance of marker effect describes the covariance structure for GBLUP better than the other weighting factors.

\section{Single-Marker Weighting Versus Group-Marker Weighting}

In addition to weighting factors, applying those to individual SNP or groups of SNP influenced genomic predictions. In fact, weighting factor $\mathrm{Wq}^{2}$ did not improve genomic predictions when using single-marker weighting. The rationale behind weighing markers when constructing a G-matrix is to describe the variances and covariances of additive genomic effects on a trait better than assuming that effects of all markers follow the same normal distribution. However, because the weighting factors were derived from estimates using a model based on a certain data set, the weights also included noise due to the uncertainty of the estimates. Therefore, the weighting factor with large uncertainty would not lead to better genomic prediction. 
Compared with single-marker weighting, groupmarker weighting, on one hand, may reduce the uncertainty of the weights but, on the other hand, dilute the real difference between marker effects. When the size of groups becomes very large, the weighted G-matrix will be similar to the original G-matrix. Compared with single-marker weightings, group-marker weightings improve prediction reliability more profoundly when using $\mathrm{Wq}^{2}$. This might reflect that the estimates of $\mathrm{q}$ had a relatively larger uncertainty. However, group-marker weighting did not have a clear advantage over singlemarker weighting in prediction reliability when using $\mathrm{Wb}^{2}$. The reason could be that using the GWAS model, only 1 marker comes into the model at a time; thus, all the markers around a causative gene will have similar estimates of effects. Accordingly, group-marker weighting will not largely differ from single-marker weighting. Using $-\log _{10}(P)$ as a weight performed slightly better than using squared estimated marker effects as weights. This could be due to the fact that $-\log _{10}(P)$ has smaller variation than the squared estimated marker effects, and larger shrinkage for extreme marker effects, thus avoiding extremely large weights on the markers that have large estimated effects with large standard error. Although group-marker weighting had a large effect on genomic prediction only when using $\mathrm{Wq}^{2}$, groupweighting reduced bias for all weighing factors. The regression coefficients of DRP on GEBV increased with increasing group size. However, when group size grew up to 30 markers, the further increase of the regression coefficient with increasing group size was very small. Moreover, group-marker weighting with a group size of 30 markers resulted in the highest reliability of GEBV for all 5 weighting factors. These suggest that this group size was appropriate for group-marker weighting in the present population. However, many factors may have an influence on the optimal group size, such as the size of the reference population, which has an effect on the uncertainty of estimated effects and variances, and population structures, which can affect LD patterns. Many potentially more reasonable methods exist for grouping markers, such as grouping markers according to the degree of LD between the markers (Castro Dias Cuyabano et al., 2012), the patterns of estimated antedependence parameters (Yang and Tempelman, 2012), the patterns of QTL intensity profile (Sillanpää and Arjas, 1998), or the patterns of estimated SNP effects or variances (Gorbach et al., 2010).

\section{Implementation of Appropriate Weights on Markers for Genomic Prediction}

The gain from the weighted G-matrix was the largest when using posterior variances estimated from the
Bayesian mixture model as a weighting factor, which led to a covariance matrix in the GBLUP model consistent with the covariance matrix in the Bayesian model, even though such a GBLUP model could not completely achieve the same prediction reliability as the Bayesian mixture model. The main reason could be that the Bayesian model estimates SNP effects and their variances simultaneously; thus, the uncertainties of all parameters in the model are taken into account in the estimation. In contrast, the GBLUP model cannot account for the uncertainties of the posterior variances that are used to construct the G-matrix. However, the Bayesian approach is a time-consuming approach and may be difficult to be used in routine genomic evaluations. An alternative solution is to store the estimated marker effects from a Bayesian model and use these estimates to predict new candidates. However, a major drawback of this strategy is that it cannot use updated phenotypic information and reference population in a timely manner. The importance of phenotypic information and size of reference population have been shown in many studies (Daetwyler et al., 2008; Goddard, 2009; Hayes et al., 2009b). In contrast, a GBLUP model with a weighted G-matrix can always use the newest data for genomic prediction, because of the low demand for computing time.

Another advantage of a weighted G-matrix is that it can be directly used in various linear mixed models, such as the single-step GBLUP model (Aguilar et al., 2010; Christensen and Lund, 2010), which predicts breeding values using information of genotyped and nongenotyped animals by integrating marker-based and pedigree-based relationship matrices into a combined relationship matrix. Wang et al. (2012) used an iterative weighting procedure in a single-step GBLUP model where the function of SNP effect estimated from the previous iteration was used as the weight to build a weighted G-matrix for prediction in the current iteration. Based on simulation data, the accuracy of predicted breeding values using this approach was 1 percentage point higher than using a BayesB model. An alternative approach is to weight a G-matrix using posterior variances of marker effects from a Bayesian variable selection model as the current study did and then use the weighted G-matrix in the single-step GBLUP model.

In the present study, the calculation of the inverse of a weighted G-matrix took about 5 min and prediction using the GBLUP model took about $3 \mathrm{~min}$ in our computing system (Intel Xeon $2.93 \mathrm{GHz}$ processor; Intel Corp., Santa Clara, CA). Many non-MCMC methods (VanRaden, 2008; Cole et al., 2009; Meuwissen et al., 2009), which are analogous to BayesA and BayesB, have been proposed and used in genomic prediction. These 
methods (often called fast BayesA and fast BayesB) can achieve prediction accuracy similar or close to that obtained by MCMC-based BayesA and BayesB while greatly reducing computing time. For a data set with a large number of genotyped animals, the fast BayesA and fast BayesB may take less time than a GBLUP model, which requires the inverse of the genomic relationship matrix. It has been shown that a GBLUP model is equivalent to a random regression SNP model (VanRaden, 2008; Strandén and Garrick, 2009). Accordingly, the gain in accuracy of genomic prediction from a weighted G-matrix using a GBLUP model would also be achieved by using a random regression SNP model with the same weighting factor to weight marker. Therefore, a random regression SNP model with appropriate weighting factor to weight marker genotypes could be a feasible alternative to GBLUP with a weighted Gmatrix when the number of genotyped animals is large. Compared with fast BayesA and BayesB methods, a random regression SNP model with weighted marker genotypes is more flexible because it can use various weighting factors (e.g., posterior variances estimated from different Bayesian variable selection models) and weighting scenarios (e.g., group-marker weighting with different definitions of groups). It can also be suggested that appropriate weighting factors, such as the posterior variance of individual marker effect from the Bayesian mixture model, can be potentially used to weight SNP markers in a single-step SNP model (Liu et al., 2014) to increase prediction accuracy.

It has been reported that the superiority of using a weighted G-matrix rather than the original G-matrix for genomic prediction increases with distant genetic relationship between validation and reference animals (Zhang et al., 2010; de los Campos et al., 2013). Many countries have used a reasonable number of juvenile bulls selected on GEBV for breeding. In the Nordic countries, a large proportion of cows mate with bulls selected on GEBV without a progeny test, and bulls used for breeding are not necessarily the sons of proven bulls. In the near future, it will be a predominant situation that sires of young candidates will not be in the reference population because they do not have the daughters' phenotypic information at the time of the candidates being selected. Therefore, the benefit from a weighted G-matrix for genomic prediction is expected to be larger than that based on current data.

Because weighting factors were derived from results of the analysis on a data set, the values of weights will change as the data are updated with more individuals available or more phenotype information for existing individuals. Therefore, weights should be updated in an appropriate interval. In fact, the GBLUP was very robust to changes in the weights applied to the G-matrix.
As shown in the current study, weighting factors derived from an analysis based on the data set collected 3 yr ago performed as well as those based on the current data. This means that a set of weights can be used up to $3 \mathrm{yr}$. Updating weighting factors once every 3 years is easily achievable in a practical breeding program.

\section{Validation of Prediction Reliability and Bias}

In the present study, reliability of genomic prediction was measured as the squared correlations between predicted breeding values and DRP and then divided by the mean reliability of DRP. This calculation of observed reliability may underestimate true reliability because it does not account for previous selection of the bulls on parent average. On the other hand, DRP in the training data were derived from current genetic evaluations. This may overestimate reliability because training data included phenotypic information from the daughters born in the same period as the test bulls. Olson et al. (2011) showed that a more appropriate way for validating genomic predictions would be using DRP derived from genetic evaluations $4 \mathrm{yr}$ ago than the current genetic evaluation. However, the validation method in the current study could be sufficient for comparing prediction methods.

In the present study, most of the estimated regression coefficients were below 1 , more serious for the 3 production traits than the other 2 traits. The prediction bias can be slightly reduced by including residual polygenic effect in the model (Liu et al., 2011; Gao et al., 2012). An additional analysis using a GBLUP model including polygenic effect showed that the deviation of regression coefficient from 1 decreased by 0.03 , averaged over the 5 traits.

\section{CONCLUSIONS}

The results from this study indicate that a GBLUP model with a G-matrix weighted using appropriate weighting factors can predict genomic breeding values with the accuracies close to those using a Bayesian variable selection model. Posterior variances of marker effects from a Bayesian mixture model are appropriate weights to construct a G-matrix for genomic prediction. A common weight on a group of about 30 markers could be a good weighting method. In a population with gradually increasing reference data, the weights can be updated once per $3 \mathrm{yr}$.

\section{ACKNOWLEDGMENTS}

This research was supported by the Center for Genomic Selection in Animals and Plants (GenSAP, Tjele, 
Denmark) funded by The Danish Council for Strategic Research (Copenhagen, Denmark).

\section{REFERENCES}

Aguilar, I., I. Misztal, D. L. Johnson, A. Legarra, S. Tsuruta, and T. J. Lawlor. 2010. Hot topic: A unified approach to utilize phenotypic, full pedigree, and genomic information for genetic evaluation of Holstein final score. J. Dairy Sci. 93:743-752.

Browning, B. L., and S. R. Browning. 2009. A unified approach to genotype imputation and haplotype-phase inference for large data sets of trios and unrelated individuals. Am. J. Hum. Genet. 84:210-223.

Castro Dias Cuyabano, B., G. Su, and M. S. Lund. 2012. Haplotype blocks built from high density marker map for genomic prediction. Page 149 in Programme \& Book of Abstracts 4th Intl. Conf. Quant. Genet., Edinburgh. In Conference Ltd., Edinburgh, UK.

Christensen, O. F., and M. S. Lund. 2010. Genomic prediction when some animals are not genotyped. Genet. Sel. Evol. 42:2.

Cole, J. B., P. M. VanRaden, J. R. O'Connell, C. P. Van Tassell, T. S. Sonstegard, R. D. Schnabel, J. F. Taylor, and G. R. Wiggans. 2009. Distribution and location of genetic effects for dairy traits. J. Dairy Sci. 92:2931-2946.

Daetwyler, H. D., B. Villanueva, and J. A. Woolliams. 2008. Accuracy of predicting the genetic risk of disease using a genome-wide approach. PLoS ONE 3:e3395.

de los Campos, G., A. I. Vazquez, R. Fernando, Y. C. Klimentidis, and D. Sorensen. 2013. Prediction of complex human traits using the genomic best linear unbiased predictor. PLoS Genet. 9:e1003608.

Erbe, M., B. J. Hayes, L. K. Matukumalli, S. Goswami, P. J. Bowman, C. M. Reich, B. A. Mason, and M. E. Goddard. 2012. Improving accuracy of genomic predictions within and between dairy cattle breeds with imputed high-density single nucleotide polymorphism panels. J. Dairy Sci. 95:4114-4129.

Gao, H., G. Su, L. Janss, Y. Zhang, and M. S. Lund. 2013. Model comparison on genomic predictions using high-density markers for different groups of bulls in the Nordic Holstein population. J. Dairy Sci. 96:4678-4687.

Gao, H., O. F. Christensen, P. Madsen, U. S. Nielsen, Y. Zhang, M. S. Lund, and G. Su. 2012. Comparison on genomic predictions using three GBLUP methods and two single-step blending methods in the Nordic Holstein population. Genet. Sel. Evol. 44:8.

Garrick, D. J., J. F. Taylor, and R. L. Fernando. 2009. Deregressing estimated breeding values and weighting information for genomic regression analyses. Genet. Sel. Evol. 41:55.

George, E. I., and R. E. McCulloch. 1993. Variable selection via Gibbs sampling. J. Am. Stat. Assoc. 88:881-889.

Goddard, M. 2009. Genomic selection: Prediction of accuracy and maximisation of long term response. Genetica 136:245-257.

Gorbach, D. M., W. Cai, J. C. M. Dekkers, J. M. Young, D. J. Garrick, R. L. Fernando, and M. F. Rothschild. 2010. Large-scale SNP association analyses of residual feed intake and its component traits in pigs. Page 265 in Proc. 9th World Congr. Genet. Appl. Livest. Prod., Leipzig, Germany. Event lab. GmbH, Leipzig, Germany.

Guo, G., M. S. Lund, Y. Zhang, and G. Su. 2010. Comparison between genomic predictions using daughter yield deviation and conventional estimated breeding value as response variables. J. Anim. Breed. Genet. 127:423-432.

Habier, D., R. L. Fernando, K. Kizilkaya, and D. J. Garrick. 2011 Extension of the Bayesian alphabet for genomic selection. BMC Bioinformatics 12:186.

Habier, D., J. Tetens, F. R. Seefried, P. Lichtner, and G. Thaller. 2010. The impact of genetic relationship information on genomic breeding values in German Holstein cattle. Genet. Sel. Evol. 42:5.

Hayes, B. J., P. J. Bowman, A. J. Chamberlain, and M. E. Goddard. 2009a. Invited review: Genomic selection in dairy cattle: Progress and challenges. J. Dairy Sci. 92:433-443.

Hayes, B. J., P. M. Visscher, and M. E. Goddard. 2009b. Increased accuracy of artificial selection by using the realized relationship matrix. Genet. Res. (Camb.) 91:47-60.
Legarra, A., C. Robert-Granié, P. Croiseau, F. Guillaume, and S. Fritz. 2011. Improved Lasso for genomic selection. Genet. Res. (Camb.) 93:77-87.

Liu, Z., M. E. Goddard, F. Reinhardt, and R. Reents. 2014. A singlestep genomic model with direct estimation of marker effects. J. Dairy Sci. 97:5833-5850.

Liu, Z., F. R. Seefried, F. Reinhardt, S. Rensing, G. Thaller, and R. Reents. 2011. Impacts of both reference population size and inclusion of a residual polygenic effect on the accuracy of genomic prediction. Genet. Sel. Evol. 43:19.

Lund, M. S., G. Sahana, D.-J. de Koning, G. Su, and Ö. Carlborg. 2009. Comparison of analyses of the QTLMAS XII common dataset. I: Genomic selection. BMC Proc. 3(Suppl. 1):S1.

Madsen, P., G. Su, R. Labouriau, and O. F. Christensen. 2010. DMUA package for analyzing multivariate mixed models. Paper 732 , Page 137 in Book of Abstracts, 9th World Congr. Genet. Appl. Livest. Prod., Leipzig, Germany. CD Comm.

Matukumalli, L. K., C. T. Lawley, R. D. Schnabel, J. F. Taylor, M. F. Allan, M. P. Heaton, J. O'Connell, S. S. Moore, T. P. L. Smith, T. S. Sonstegard, and C. P. Van Tassell. 2009. Development and characterization of a high density SNP genotyping assay for cattle. PLoS ONE 4:e5350.

Meuwissen, T., and M. Goddard. 2010. Accurate prediction of genetic values for complex traits by whole-genome resequencing. Genetics 185:623-631.

Meuwissen, T. H. E., B. J. Hayes, and M. E. Goddard. 2001. Prediction of total genetic value using genome-wide dense marker maps. Genetics 157:1819-1829.

Meuwissen, T. H. E., T. R. Solberg, R. Shepherd, and J. A. Woolliams. 2009. A fast algorithm for BayesB type of prediction of genomewide estimates of genetic value. Genet. Sel. Evol. 41:2.

Olson, K. M., P. M. VanRaden, M. E. Tooker, and T. A. Cooper. 2011. Differences among methods to validate genomic evaluations for dairy cattle. J. Dairy Sci. 94:2613-2620.

Sillanpää, M. J., and E. Arjas. 1998. Bayesian mapping of multiple quantitative trait loci from incomplete inbred line cross data. Genetics 148:1373-1388.

Strandén, I., and D. J. Garrick. 2009. Technical note: Derivation of equivalent computing algorithms for genomic predictions and reliabilities of animal merit. J. Dairy Sci. 92:2971-2975.

Su, G., R. F. Brøndum, P. Ma, B. Guldbrandtsen, G. R. Aamand, and M. S. Lund. 2012a. Comparison of genomic predictions using medium-density $(\sim 54,000)$ and high-density $(\sim 777,000)$ single nucleotide polymorphism marker panels in Nordic Holstein and Red Dairy Cattle populations. J. Dairy Sci. 95:4657-4665.

Su, G., B. Guldbrandtsen, V. R. Gregersen, and M. S. Lund. 2010. Preliminary investigation on reliability of genomic estimated breeding values in the Danish Holstein population. J. Dairy Sci. 93:1175-1183.

Su, G., P. Madsen, U. S. Nielsen, E. A. Mäntysaari, G. P. Aamand, O. F. Christensen, and M. S. Lund. 2012b. Genomic prediction for Nordic Red Cattle using one-step and selection index blending. J. Dairy Sci. 95:909-917.

VanRaden, P. M. 2008. Efficient methods to compute genomic predictions. J. Dairy Sci. 91:4414-4423.

Wang, H., I. Misztal, I. Aguilar, A. Legarra, and W. M. Muir. 2012. Genome-wide association mapping including phenotypes from relatives without genotypes. Genet. Res. (Camb.) 94:73-83.

Yang, W., and R. J. Tempelman. 2012. A Bayesian antedependence model for whole genome prediction. Genetics 190:1491-1501.

Zhang, Z., J. Liu, X. Ding, P. Bijma, D.-J. de Koning, and Q. Zhang. 2010. Best linear unbiased prediction of genomic breeding values using a trait-specific marker-derived relationship matrix. PLoS ONE 5:e12648.

Zhou, L., M. S. Lund, Y. Wang, and G. Su. 2014. Genomic predictions across Nordic Holstein and Nordic Red using the genomic best linear unbiased prediction model with different genomic relationship matrices. J. Anim. Breed. Genet. 131:249-257. http://dx.doi. org/10.1111/jbg.12089. 


\section{APPENDIX}

Reliabilities and unbiasedness of genomic predictions for each trait using various weighted G-matrices are given in Tables A1, A2, A3, and A4. The weighting factors were $\mathrm{Wb}^{2}, \mathrm{Wq}^{2}, \mathrm{WP}_{\mathrm{b}}, \mathrm{WP}_{\mathrm{q}}$, and $\mathrm{WV}_{\mathrm{q}}$. As shown in Table A1, no single weighting factor was more accurate than the others for all traits when using single-marker weighting. However, weighting factor $\mathrm{Wq}^{2}$ resulted in the lowest reliability for milk, protein, and mastitis, $\mathrm{WP}_{\mathrm{q}}$ for fat, and $\mathrm{Wb}^{2}$ for fertility. When using group-marker weighting with group size of 30 markers, $\mathrm{WV}_{\mathrm{q}}$ led to highest reliability for all traits (Table A2). Although $\mathrm{Wq}^{2}$ got the largest benefit of group-marker weighting, this weighting factor still led to the lowest reliability for protein and mastitis. In addition, $\mathrm{Wq}^{2}$ resulted in the lowest regression coefficients (i.e., the highest inflation) for all traits when using single-marker weighting (Table A3), and for protein, fertility, and mastitis when using group-marker weighting (Table A4).

Table A1. Reliability of genomic prediction using genomic relationship matrices (G-matrices) with various weighted G-matrices by single-marker weighting ${ }^{1}$

\begin{tabular}{lccccc}
\hline Item & $\mathrm{WV}_{\mathrm{q}}$ & $\mathrm{Wq}^{2}$ & $\mathrm{WP}_{\mathrm{q}}$ & $\mathrm{Wb}^{2}$ & $\mathrm{WP}_{\mathrm{b}}$ \\
\hline Trait & & & & & \\
Milk & 0.502 & 0.486 & 0.505 & 0.500 & 0.502 \\
Fat & 0.491 & 0.481 & 0.463 & 0.490 & 0.494 \\
Protein & 0.477 & 0.456 & 0.466 & 0.481 & 0.476 \\
Fertility & 0.449 & 0.425 & 0.452 & 0.418 & 0.421 \\
Mastitis & 0.400 & 0.381 & 0.397 & 0.393 & 0.395 \\
Mean & 0.464 & 0.446 & 0.457 & 0.456 & 0.458 \\
\hline
\end{tabular}

${ }^{1} \mathrm{WV}_{\mathrm{q}}=$ posterior variance of individual marker effect from the Bayesian mixture model; $\mathrm{Wq}^{2}=$ square of the marker effect estimated from the Bayesian mixture model; $\mathrm{WP}_{\mathrm{q}}=-\log _{10}(P$-value $)$ from a $t$-test for SNP effect estimated from the Bayesian mixture model; $\mathrm{Wb}^{2}=$ square of marker effect estimated from the genome-wide association study (GWAS) model; $\mathrm{WP}_{\mathrm{b}}=-\log _{10}(P$-value $)$ from a $t$-test for SNP effect estimated from the GWAS model.

Table A2. Reliability of genomic prediction using genomic relationship matrices (G-matrices) with various weighted G-matrices by group-marker weighting with group size of 30 markers

\begin{tabular}{lccccc}
\hline Item & $\mathrm{WV}_{\mathrm{q}}$ & $\mathrm{Wq}^{2}$ & $\mathrm{WP}_{\mathrm{q}}$ & $\mathrm{Wb}^{2}$ & $\mathrm{WP}_{\mathrm{b}}$ \\
\hline Trait & & & & & \\
Milk & 0.511 & 0.500 & 0.504 & 0.498 & 0.501 \\
Fat & 0.505 & 0.493 & 0.480 & 0.499 & 0.500 \\
Protein & 0.472 & 0.465 & 0.466 & 0.461 & 0.461 \\
Fertility & 0.449 & 0.437 & 0.449 & 0.432 & 0.439 \\
Mastitis & 0.401 & 0.385 & 0.399 & 0.397 & 0.397 \\
Mean & 0.468 & 0.456 & 0.460 & 0.457 & 0.460 \\
\hline
\end{tabular}

${ }^{1} \mathrm{WV}_{\mathrm{q}}=$ posterior variance of individual marker effect from the Bayesian mixture model; $\mathrm{Wq}^{2}=$ square of the marker effect estimated from the Bayesian mixture model; $\mathrm{WP}_{\mathrm{q}}=-\log _{10}(P$-value $)$ from a $t$-test for SNP effect estimated from the Bayesian mixture model; $\mathrm{Wb}^{2}=$ square of marker effect estimated from the genome-wide association study (GWAS) model; $\mathrm{WP}_{\mathrm{b}}=-\log _{10}(P$-value) from a $t$-test for SNP effect estimated from the GWAS model.

Table A3. Regression of deregressed proof on genomic prediction using genomic relationship matrices (G-matrices) with various weighted G-matrices by single-marker weighting

\begin{tabular}{lccccc}
\hline Item & $\mathrm{WV}_{\mathrm{q}}$ & $\mathrm{Wq}^{2}$ & $\mathrm{WP}_{\mathrm{q}}$ & $\mathrm{Wb}^{2}$ & $\mathrm{WP}_{\mathrm{b}}$ \\
\hline Trait & & & & & \\
Milk & 0.825 & 0.787 & 0.829 & 0.864 & 0.863 \\
Fat & 0.759 & 0.736 & 0.784 & 0.807 & 0.805 \\
Protein & 0.792 & 0.728 & 0.776 & 0.811 & 0.805 \\
Fertility & 0.938 & 0.801 & 0.894 & 0.860 & 0.861 \\
Mastitis & 0.846 & 0.754 & 0.826 & 0.848 & 0.850 \\
Mean & 0.832 & 0.761 & 0.822 & 0.838 & 0.837 \\
\hline
\end{tabular}

${ }^{1} \mathrm{WV}_{\mathrm{q}}=$ posterior variance of individual marker effect from the Bayesian mixture model; $\mathrm{Wq}^{2}=$ square of the marker effect estimated from the Bayesian mixture model; $\mathrm{WP}_{\mathrm{q}}=-\log _{10}(P$-value $)$ from a $t$-test for SNP effect estimated from the Bayesian mixture model; $\mathrm{Wb}^{2}=$ square of marker effect estimated from the genome-wide association study (GWAS) model; $\mathrm{WP}_{\mathrm{b}}=-\log _{10}(P$-value $)$ from a $t$-test for SNP effect estimated from the GWAS model. 
Table A4. Regression of deregressed proof on genomic prediction using genomic relationship matrices (G-matrices) with various weighted G-matrices by group-marker weighting with group size of 30 markers

\begin{tabular}{lccccc}
\hline Item & $\mathrm{WV}_{\mathrm{q}}$ & $\mathrm{Wq}^{2}$ & $\mathrm{WP}_{\mathrm{q}}$ & $\mathrm{Wb}^{2}$ & $\mathrm{WP}_{\mathrm{b}}$ \\
\hline Trait & & & & & \\
Milk & 0.869 & 0.887 & 0.874 & 0.865 & 0.869 \\
Fat & 0.816 & 0.836 & 0.840 & 0.818 & 0.821 \\
Protein & 0.823 & 0.792 & 0.816 & 0.810 & 0.813 \\
Fertility & 0.980 & 0.910 & 0.968 & 0.933 & 0.943 \\
Mastitis & 0.901 & 0.837 & 0.895 & 0.884 & 0.883 \\
Mean & 0.878 & 0.852 & 0.879 & 0.862 & 0.866 \\
\hline
\end{tabular}

${ }^{1} \mathrm{WV}_{\mathrm{q}}=$ posterior variance of individual marker effect from the Bayesian mixture model; $\mathrm{Wq}^{2}=$ square of the marker effect estimated from the Bayesian mixture model; $\mathrm{WP}_{\mathrm{q}}=-\log _{10}(P$-value $)$ from a $t$-test for SNP effect estimated from the Bayesian mixture model; $\mathrm{Wb}^{2}=$ square of marker effect estimated from the genome-wide association study (GWAS) model; $\mathrm{WP}_{\mathrm{b}}=-\log _{10}(P$-value $)$ from a $t$-test for SNP effect estimated from the GWAS model. 